\title{
The Impact of Heterogeneity in Purchase Timing and Price Responsiveness on Estimates of Sticker Shock Effects
}

\author{
Kwangpil Chang • S. Siddarth • Charles B. Weinberg \\ J. D. Power and Associates, 30401 Agoura Road, Agoura Hills, California 91301 \\ Marshall School of Business, University of Southern California, Los Angeles, California 90089-1421 \\ siddarth@bus.usc.edu \\ Faculty of Commerce, University of British Columbia, Vancouver, British Columbia V6T 1Z2, Canada, \\ charles.b.weinberg@ubc.ca
}

\begin{abstract}
The notion that individuals have an internal reference price against which they compare observed prices is well supported by several psychological theories. Empirically, several papers in the marketing literature, employing scanner panel data, have modeled the impact of reference prices on brand choice via the sticker shock formulation, in which consumers evaluate choice alternatives using differences between shelf prices and reference prices. Most of the studies reported thus far have not accounted for heterogeneity in price response among consumers and have typically imputed reference prices from the shelf prices of brands that a consumer is supposed to have "observed" on previous purchases in the category. Since category marketing activity can differentially affect the purchase timing of households, we argue that this measure of reference price may follow certain systematic patterns across consumers and, when combined with unaccounted for price response heterogeneity, may result in a spurious sticker shock effect. Specifically, we show that estimates of sticker shock are biased upward if households that are price-sensitive in the brand choice decision are also more responsive to category promotion activity in their purchase timing decision.

We discuss some general conditions under which the bias occurs and conduct a simulation experiment to confirm our specific hypotheses. Our simulation results show that changes in purchase timing are a critical determinant of the bias in the sticker shock effect. We also show that unaccounted for price response heterogeneity can in itself result
\end{abstract}

in a biased sticker shock parameter; however, this requires very large differences in price sensitivities across consumers, far greater than what is normally observed.

We develop a hierarchical Bayes version of the nested logit model, which models heterogeneity via individual-level parameters in a continuous random effects framework. We estimate the model on scanner panel data from the yogurt and ketchup categories. We find, in both categories, that the $95 \%$ probability interval of the posterior distribution of the mean sticker shock coefficient contains the value zero. Therefore, at least for the data used in this study, there is no evidence for the sticker shock effect at the aggregate level. In contrast, the corresponding coefficient from a standard model (which ignores this heterogeneity) is highly significant and supports the existence of a (possibly spurious) sticker shock effect. Consistent with our explanation of the underlying cause of the bias, households that are more price-sensitive in the choice decision are also found to be more responsive to category promotion activity in their decision to purchase in the category.

The results highlight the measurement problems associated with imputing reference prices from past prices. Since the frequency, duration, and price level of a retailer's promotional program depend on its size and prevalence, accurate estimates of the sticker shock effect are essential for formulating optimal promotion strategies. An adequate accounting of consumer heterogeneity is critical to this effort. (Hierarchical Bayes Approach; Choice Models; Buyer Behavior; Reference Price) 
THE IMPACT OF HETEROGENEITY IN PURCHASE TIMING AND PRICE

RESPONSIVENESS ON ESTIMATES OF STICKER SHOCK EFFECTS

\section{Introduction}

The notion that individuals have an internal reference price against which they compare observed prices is well supported by several psychological theories, notably the Adaptation-Level Theory (Helson 1964), Assimilation-Contrast Theory (Sherif et al. 1958), and Prospect Theory (Kahneman and Tversky 1979). Several researchers (e.g., Winer 1986, Lattin and Bucklin 1989) have incorporated the impact of reference prices on consumers using disaggregate models of brand choice behavior. The most common approach used to capture the hypothesized impact of reference price in empirical brand choice models is to include in the utility function of a logit choice model, a variable, commonly referred to as the "sticker shock" term (Winer 1986), that represents the difference between a brand's reference price and its current shelf price. A positive coefficient for the associated parameter is interpreted as evidence for the sticker shock effect. These models have also been extended to incorporate more sophisticated responses to reference prices, including asymmetries in response to gains and losses (e.g., Kalwani et al. 1990) and zones of indifference around reference prices (Kalyanaram and Little 1994).

Previous empirical applications of the sticker shock model share three common features. First, model estimation has not accounted for heterogeneity in price response among consumers (Briesch et al. 1997 is an exception). Second, because reference price is a latent construct that is not directly observable, it has been imputed from the price series in the data. Finally, the imputed reference price measure has been based on those trips on which consumers actually made a purchase in the category. As a result, the imputed measure directly incorporates the impact of pricing and promotion activity on the purchase timing decision-a behavior that has been extensively studied in the marketing literature, e.g., Gupta (1988), Jain and Vilcassim (1991), Bucklin and Gupta (1992).

In this paper, we show that unaccounted-for price response heterogeneity can bias estimates of the sticker shock parameter if consumers who are more pricesensitive in the brand choice decision are also more responsive to category marketing activity in the purchase timing decision, a relationship supported by some previous empirical findings (Bucklin and Gupta
1992, Bucklin et al. 1998). We note that our study and results do not dispute the theoretical underpinnings or the existence of reference prices, but highlight the problems associated with the manner in which they have been measured, and reference price effects estimated, in empirical brand choice models.

The findings from our research parallel those of Bell and Lattin (1998), who also show that unaccounted-for price response heterogeneity can bias estimates of reference price effects. However, the two studies also differ in several important ways. First, while Bell and Lattin (1998) analyze the less commonly used reference dependent formulation (Hardie et al. 1993), that employs a single category reference price, we study the more widely used sticker shock model in which reference prices are brand-specific. Second, while they focus exclusively on price response heterogeneity, we also analyze the important role played by purchase timing heterogeneity. Third, in contrast to their work, we conduct an extensive simulation to study the conditions under which the bias occurs.

Under the conditions stated previously, pricesensitive households are more likely to purchase in the category when it is more "attractive," i.e., when one or more brands is on promotion, while price-insensitive households are less likely to change the timing of their purchases because of promotional activity. Therefore, observed across-brand shelf prices are lower for pricesensitive households than for insensitive households, and reference price measures imputed from prices on previous purchase occasions also display the same pattern. Because this pattern of reference prices mimics the unaccounted for response heterogeneity, it can result in a spuriously significant sticker shock parameter.

The biasing effects of unobserved heterogeneity on parameter estimates in models of choice and purchase incidence has been previously highlighted by several researchers in marketing. For example, in the brand choice literature, Gonul and Srinivasan (1991) show that a simple heterogeneity specification tends to understate the impact that marketing variables have on brand choice behavior. Chintagunta (1992) reports that ignoring similarities across choice alternatives results in incorrect estimates for marketing mix effects. Similarly, the results of Jain and Vilcassim (1991) and Chintagunta (1993) demonstrate the critical impact 
that unobserved heterogeneity can have on parameter estimates in models of category purchase decisions.

Recently, researchers have proposed several hierarchical Bayes models, based on both probit (McCulloch and Rossi 1994, Rossi et al. 1996), and logit choice probabilities (Allenby and Lenk 1994, Arora et al. 1998, Allenby et al. 1998), which flexibly incorporate consumer response heterogeneity, and have documented the enormous variations in household priceresponsiveness in frequently purchased package goods categories. This empirical finding makes it more likely that the reference price measurement problem described above exists, and results in incorrect estimates of sticker shock effects. Based on this background, and our desire to investigate brand choice and purchase timing decisions together, we formulate a hierarchical Bayes version of the nested logit model that incorporates response heterogeneity via householdlevel parameters in a continuous random effects framework.

We estimate this model on scanner panel data from the yogurt and ketchup categories and find that, in both categories, the $95 \%$ probability interval of the posterior distribution of the mean sticker shock coefficient contains the value zero. Therefore, at least for these data sets, the results do not support the existence of a sticker shock effect as operationalized in previous research. In contrast, the sticker shock coefficient obtained from a standard nested logit model, which does not account for price response heterogeneity, is statistically significant. In both categories, consistent with our explanation of the underlying cause of the spurious effects, we find that households that are pricesensitive in the choice decision are also more sensitive to category promotion activity in their purchase incidence decision.

The remainder of the paper is organized as follows. Section 2 presents a stylized model to describe the mechanism of parameter bias. Section 3 describes both the standard and hierarchical Bayes nested logit models used in the estimation. Section 4 describes a simulation study that establishes the conditions under which the bias occurs. Section 5 discusses the empirical application and results from our analysis of scanner panel data. Section 6 summarizes our conclusions and provides directions for future research.

\section{Understanding the Mechanism of Sticker Shock Bias}

We begin with a general model of choice that includes a sticker shock effect. Because we are particularly interested in examining the conditions that can produce spurious estimates of the sticker shock coefficient, we consider a special case of this model in which reference prices do not influence brand choice. Thus, assuming that the "true" model underlying brand choice behavior does not include a sticker shock effect, we analyze the consequences of estimating a "misspecified" model, which ignores price response heterogeneity and (incorrectly) includes a sticker shock term. We discuss the conditions under which the sticker shock coefficient obtained from this misspecified model can be spuriously significant.

\subsection{A General Model of Sticker Shock Choice}

The deterministic component of household $h$ 's utility for brand $i$ on occasion $t$ is given as:

$$
\begin{aligned}
U_{i t}^{h}= & \alpha_{i}+\left(\beta_{\mathrm{mp}}+\delta^{h}\right) \text { PRICE }_{i t} \\
& +\left(\beta_{\mathrm{mrp}}+\varepsilon^{h}\right)\left(\text { RPRICE }_{i t}^{h}-\text { PRICE }_{i t}\right),
\end{aligned}
$$

where $\alpha_{i}$ is a brand intercept, PRICE $E_{i t}$ is the price, and $\mathrm{RPRICE}_{i t}^{h}$ is the reference price, for household $h$ brand $i$ on occasion $t$. Also, $\beta_{\mathrm{mp}}$ and $\beta_{\mathrm{mrp}}$ are the mean price and reference price parameters, respectively, and $\delta^{h}$ and $\epsilon^{h}$ are household-specific components of these parameters, distributed $N\left(0, \sigma_{\delta}^{2}\right)$ and $N\left(0, \sigma_{\varepsilon}^{2}\right)$, respectively, across households. To illustrate the logic underlying the bias more clearly, other terms are omitted from the utility specification.

\subsection{Description of the True Model}

We analyze a special case of Equation (1) in which households are assumed not to respond to reference prices, i.e., $\beta_{\mathrm{mrp}}=0$, and $\sigma_{\varepsilon}^{2}=0$. The deterministic component of household $h^{\prime}$ s utility for brand $i$ on occasion $t$ is given by:

$$
U_{i t}^{h}=\alpha_{i}+\left(\beta_{\mathrm{mp}}+\delta^{h}\right) \mathrm{PRICE}_{i t} .
$$

\subsection{Analysis of the Misspecified Model}

We take the position of an analyst who (incorrectly) ignores differences in price sensitivity across households, and captures price responsiveness by the single 
common price parameter, $\beta_{c}$. An additional misspecification error is introduced by including in each brand's utility function a sticker shock term-the difference between its reference price and its actual price. The resulting utility function, similar to that used in previous research, is:

$$
U_{i t}^{h}=\alpha_{i}+\beta_{c} \text { PRICE }_{i t}+\beta_{\text {rp }}\left(\text { RPRICE }_{i t}^{h}-\text { PRICE }_{i t}\right),
$$

which may also be rewritten as,

$$
U_{i t}^{h}=\alpha_{i}+\left(\beta_{c}-\beta_{\mathrm{rp}}\right) \mathrm{PRICE}_{i t}+\beta_{r p} \text { RPRICE }_{i t}^{h} \text {. }
$$

An estimate of $\beta_{\mathrm{rp}}>0$ would (incorrectly) imply that consumers experience sticker shock in the brand choice decision. We now briefly describe commonly used approaches to impute reference prices from past prices, and we show how these measures can result in a spuriously significant estimate of $\beta_{\mathrm{rp}}$.

Measuring Reference Price. Because reference price is an internal, latent construct, it has to be imputed from the prices paid (or observed) by the consumer on previous shopping occasions. The standard approach has been to operationalize reference price as a function of brand prices "observed" by the consumer on those trips on which a category purchase actually occurred.

Kalyanaram and Little (1994) use an exponentially smoothed measure of past shelf prices encountered by a consumer and estimate the smoothing parameter to be 0.82 , implying that the most recent price has the maximum influence on reference price. Kalwani et al. (1990) summarize the impact of the previous five prices and find the most recent price to be weighted most heavily. Rajendran and Tellis (1994) use the weighted mean of the previous three prices with the most recent price weighted most heavily as a brandspecific temporal reference price. ${ }^{1}$ Mayhew and Winer's (1992) exponential smoothing of past prices resulted in an optimal smoothing constant of one and led them to set reference price equal to that observed

\footnotetext{
${ }^{1}$ In addition, they introduce deviations from a category-specific contextual reference price, $\mathrm{Rc}_{t}$, operationalized, alternatively, as the lowest, mean, and highest of the prices observed on the current purchase occasion. Since $\mathrm{Rc}_{t}$ is common across brands, it drops out of the logit equation. Thus, in their estimated model, an actual price and a brand-specific temporal reference price remain.
}

on the previous purchase occasion, i.e., $\mathrm{RPRICE}_{i t}^{h}=$ PRICE $_{i(t-1)}$. A similar approach is also used by Bell and Bucklin (1999).

Consistent with these findings and with earlier operationalizations of the reference price construct, we set the reference price of a brand equal to its price on the previous purchase occasion. While this definition has the added advantage of simplifying our exposition in this part of the paper, we note that the logic that we describe carries over to more complicated measures of reference price which are based on the past prices that the consumer is supposed to have actually paid or observed. Moreover, in our empirical analysis, we also re-estimate our model using a reference price measure that exponentially smoothes past prices, and we find a statistically insignificant sticker shock effect after properly accounting for response heterogeneity.

Role of Purchase Timing. A significant body of previous research has investigated the impact of marketing activity on the purchase timing decision (Gupta 1988, Jain and Vilcassim 1991, Helsen and Schmittlein 1993). Bucklin and Gupta (1992) and Bucklin et al. (1998) provide some empirical evidence that shows that price-sensitive consumers have a greater tendency to change purchase timing to coincide with promotional activity than price-insensitive consumers.

This empirical finding suggests that price-sensitive consumers may be more likely to make a purchase when the category is "attractive," i.e., when one or more brands is on promotion. Price-insensitive consumers, on the other hand, will be less likely to change the timing of their purchases because of promotional activity, hence a smaller proportion of their purchases will coincide with promotional activity. The observed across-brand shelf prices and, consequently, the imputed reference prices for the next purchase occasion, will be lower for the price-sensitive consumers than for the price-insensitive consumers.

A comparison of Equations (2) and (4) shows that a positive value for the sticker shock coefficient, $\beta_{\mathrm{rp}}$, can compensate for omitted response heterogeneity. In other words, a positive $\beta_{\mathrm{rp}}$ makes $\left(\beta_{c}-\beta_{\mathrm{rp}}\right)$ more negative, providing a better representation of the behavior of the price-sensitive households. Moreover, this compensating mechanism also permits $\beta_{c}$ to take on a value 
which better represents the less price-sensitive households. Overall, the presence of $\beta_{\mathrm{rp}}$ makes the model more flexible in representing the underlying price heterogeneity than a standard logit model $-\beta_{c}$ captures the response of households that are price-insensitive, while $\beta_{c}$ and $\beta_{\mathrm{rp}}$ together capture the response of those who are price-sensitive.

A similar effect could also be produced by large variations in brand choice price sensitivity, i.e., when $\sigma_{\delta}^{2}$ is very large. A possible outcome under this condition is that lower priced brands may be exclusively purchased by the extremely price sensitive households, and the higher priced brands by the price-insensitive households. This outcome could result in the reference price variable and the omitted variable being correlated, even without heterogeneity in purchase timing, resulting in a spurious sticker shock parameter. In our simulation, we study how the range of price responsiveness impacts the bias in the absence of differences in purchase timing across the segments. We find that only very large differences in responsiveness result in significant bias and that the implied range of price elasticities is much larger than has been observed in practice (Tellis 1988). In contrast, we find that even moderate differences in price responsiveness, when combined with heterogeneity in purchase timing, can result in a biased sticker shock parameter.

\section{Estimated Models}

Previous research has primarily analyzed the impact of the sticker shock term via a multinomial logit model of brand choice behavior. Because it is important to capture fully any theoretical argument in the empirical model specification, and because we argue that aspects of purchase incidence behavior can play an important role in biasing the sticker shock coefficient, we incorporate the multinomial brand choice model within a nested logit model of purchase incidence. In the empirical application, the use of this formulation enables us to confirm that the hypothesized relationship between brand choice and purchase incidence behavior is consistent with our theoretical argument. Three versions of these models are estimated.

\subsection{Nested Logit (NL) Model}

In this baseline model, consumers choose a brand without relying on an internal reference price and share the same response parameters. The NL model specifies the probability of brand choice for household $h$ on occasion $t$, conditional on a category purchase, as:

$$
P_{t}^{h}(i \mid \text { inc })=\frac{\exp \left(U_{i t}^{h}\right)}{\Sigma_{k} \exp \left(U_{k t}^{h}\right)} .
$$

$U_{i t}^{h}$ denotes the deterministic component of utility for each alternative $i$ that is a function of both householdspecific, and marketing mix variables, with an associated parameter vector $\boldsymbol{\beta}$.

The probability of purchase incidence for household $h$, conditional on a store visit at time $t$, is given by (e.g., Bucklin and Gupta 1992):

$$
P_{t}^{h}(\text { inc })=\frac{\exp \left(V_{t}^{h}\right)}{1+\exp \left(V_{t}^{h}\right)},
$$

where $V_{t}^{h}$ denotes the deterministic component of utility for purchase incidence, which is a function of household and marketing mix variables and associated parameter vector $\gamma$.

\subsection{Nested Logit Sticker Shock (NL-ST) Model}

The NL-ST model calls for augmenting the NL brand choice utility function with the sticker shock term, i.e., the difference between a brand's reference price and its shelf price. A brand's reference price is assumed to be its price on the last occasion on which the household made a purchase in the category. Brand choice and purchase incidence probabilities are given by Equations (5) and (6), respectively. The choice and incidence parameters, $\boldsymbol{\beta}$ and $\gamma$, respectively, for both the NL and the NL-ST models are obtained by maximum likelihood.

\subsection{Hierarchical Bayes Sticker Shock (HB-ST) Model}

We also formulate a hierarchical Bayes model of incidence and sticker shock choice in which heterogeneity in choice and incidence parameters is modeled in three stages. In the first stage, the brand choice and purchase incidence models are written with household-specific parameters. In the second stage, the prior (population) distribution over the household-specific parameter vectors, $\boldsymbol{\theta}^{h}=\left(\boldsymbol{\beta}^{h}, \boldsymbol{\gamma}^{h}\right)$, is specified as multivariate normal, i.e., $\boldsymbol{\theta}^{h} \sim \operatorname{MVN}(\boldsymbol{\mu}, \boldsymbol{\Sigma})$. The covariance matrix of household parameter vectors, $\boldsymbol{\Sigma}$, captures the unobserved source of heterogeneity across households. 
Since the fully Bayesian approach requires priors over unknown quantities in the model, the third stage of the model specifies hyper-priors over the parameters of the population distribution, $\boldsymbol{\mu}$ and $\boldsymbol{\Sigma}$. We assume a multivariate normal hyper-prior over $\boldsymbol{\mu}, \operatorname{MVN}(\boldsymbol{\eta}, \mathbf{C})$, and a Wishart prior over $\Sigma, W\left[(\rho \mathbf{R})^{-1}, \rho\right]$, following the standard approach suggested in the statistics literature (Gelfand and Smith 1990, Gelfand et al. 1990). Details on each prior are provided in Appendix A.

This hierarchical Bayes set up involves evaluating high-order multivariate normal integrals required in formulating the posterior density, making it practically impossible to use conventional maximum likelihood estimation techniques. We circumvent this problem by using sampling-based Bayesian approaches that build upon Markov Chain Monte Carlo (MCMC) methods (Gelfand and Smith 1990, Chib and Greenberg 1995, Allenby et al. 1998). Using the sampling-based approach permits one to account properly for uncertainty in inferences and to make exact (as opposed to asymptotic) inference possible with any desired precision (McCulloch and Rossi 1994).

Sampling from the joint posterior distribution is achieved by sampling from the full conditional distributions, known as substitution sampling (Details on the full conditional distributions for model parameters are provided in Appendix B). If all the full conditional distributions are known (i.e., known conjugate distributions such as a normal and a Wishart), then substitution sampling reduces to a procedure known as Gibbs Sampling (Geman and Geman 1984, Gelfand and Smith 1990). If the full conditional distributions are not completely known (i.e., known only up to a normalizing constant), then a Metropolis-Hastings step is used (Tierney 1994, Chib and Greenberg 1995). For the HB-ST model, the $(m+1)$ th step of the substitution sampling involves generating the following draws:

(a) Generate $\boldsymbol{\theta}^{\mathbf{h}}$ draws from $p\left(\boldsymbol{\theta}^{\mathbf{h}(m+1)} \mid \mathbf{\Sigma}^{(m)}, \boldsymbol{\mu}^{(m)}\right)$ for $h$ $=1$ to $H$, using a Metropolis-Hastings algorithm.

(b) Generate a $\boldsymbol{\mu}$ draw from $p\left(\boldsymbol{\mu}^{(m+1)} \mid\left\{\boldsymbol{\theta}^{h(m)}\right\}, \boldsymbol{\Sigma}^{(m)}, \boldsymbol{\eta}\right.$, C), using a Gibbs sampler.

(c) Generate a $\boldsymbol{\Sigma}^{-1}$ draw from $p\left(\boldsymbol{\Sigma}^{-1(m+1)} \mid\left\{\boldsymbol{\theta}^{h(m)}\right\}\right.$, $\left.\mu^{(m)}, \rho, \mathbf{R}\right)$, using a Gibbs sampler.

This sequence of draws generates a Markov chain whose stationary distribution is the joint posterior distribution of all unknown parameters. The initial draws (1st to $m$ th) from the chain reflect a transient period in which the chain has not converged to the equilibrium distribution, and is therefore discarded. A sample of draws obtained after convergence is used to make posterior inferences about model parameters. It should be noted that this sampling-based procedure generates the entire posterior distribution of interest, rather than just a point estimate. Given that we expect substantial uncertainty in inferring household parameters with only a small number of observations, it is critical that we should also measure properly the uncertainty of estimates (Rossi et al. 1996).

In our application, the substitution sampler was run for 15,000 iterations and convergence was ensured by monitoring the time-series of the draws. We chose a burn-in length of 10,000 iterations and retained every fifth iteration ("thinning the chain," Raftery and Lewis 1995) of the remaining 5,000 iterations to reduce the serial correlation of the sampled draws. Therefore, 1,000 draws from the posterior distribution of each parameter were used to make inferences.

\section{Simulation Study}

We conduct a simulation experiment to study the conditions under which the unaccounted price response heterogeneity produces a biased sticker shock coefficient. The simulation varies two factors, each with two levels. The factors are price response heterogeneity (moderate or extreme) and purchase timing heterogeneity (present or absent). In each experimental cell, we simulate choices of hypothetical panelists corresponding to the true model of Equation (2), i.e., assuming that consumers do not respond to reference prices. The "plain" Nested Logit (NL) model, the corresponding sticker shock model (NL-ST) and a Hierarchical Bayes version of this model (HB-ST) are then estimated on the simulated data.

Based on our earlier analysis, we make three predictions about the parameters obtained from the misspecified NL-ST model corresponding to Equation (3). First, the parameter $\beta_{r p}$ will be spuriously positive and significant when heterogeneity in purchase timing is present across households, whether price response heterogeneity is moderate or extreme. Second, $\beta_{r p}$ will not 
be biased when purchase timing heterogeneity is absent if price response heterogeneity is moderate. Third, the common price parameter $\beta_{c}$ will be smaller in absolute value than the estimate obtained from the NL model. In addition, we expect that the HB-ST model, which appropriately accounts for price heterogeneity, will not produce a spuriously significant sticker shock coefficient.

\subsection{Data Generation}

Category purchases were simulated via a nested logit, and brand choice decisions via a multinomial logit, for 400 hypothetical households. Household-level price response parameters in the brand choice decision were drawn from a normal distribution. Each household encountered 60 different store environments, i.e., made 60 shopping trips. We generated 60 prices for each of three hypothetical brands of which the first two, A and $\mathrm{B}$, were assumed to be national brands while the third $\mathrm{C}$, was a lower-priced generic brand. Price and promotional levels are shown in Table 1.

On each shopping trip the utility of each brand was calculated based on the utility functions shown in Table 1 . The brand-specific constants were chosen to reflect the higher preference for the national brands. The decision to purchase in the category was modeled using a nested logit model in which the incidence utility was specified as a function of a constant and the category value $(\mathrm{CV})$ as follows:

$$
V^{h}=a^{h}+\beta_{i n c}^{h} \mathrm{CV}^{h},
$$

where $\beta_{i n c}^{h}$ is the $\mathrm{CV}$ response parameter for household $h$, and $\mathrm{CV}^{h}$ for each trip is calculated as $\ln \left[\exp \left(U_{A}^{h}\right)+\right.$ $\left.\exp \left(U_{B}^{h}\right)+\exp \left(U_{C}^{h}\right)\right]$. The parameter $a^{h}$ was chosen such that the overall incidence probability for each household resulted in approximately 15 category purchases. The probability of category purchase is given by:

$$
P^{h}(\text { inc })=\frac{\exp \left(V^{h}\right)}{1+\exp \left(V^{h}\right)} .
$$

If a draw from the binomial vector $P^{h}($ inc) resulted in a predicted category purchase, then the brand choice decision was simulated using the multinomial logit choice probability vector. If the draw resulted in a nonpurchase, we repeated the above steps for the next store environment. Thus, the resulting data set contained about 6,000 $(15 \times 400)$ "choice" observations and 18,000(45 $\times 400)$ "nonpurchase" occasions.

Specifying Parameter Values for Choice and Incidence Decision.

Choice: In a meta-analysis of 367 price elasticities, Tellis (1988) reports elasticities in the range from +2 to -10 with a vast majority falling in the range -1 to -9 . Based on these values, for the moderate price response heterogeneity condition, $\beta^{h}$ was drawn from a $\operatorname{Normal}\left(-3,1^{2}\right)$ distribution, which implies that $95 \%$ of elasticities lie in range from -1.25 to -10.30 and that the market-level elasticity is -4.13 . The chosen range of elasticities is also consistent with that implied by the price parameters estimated by Arora et al. (1998). For the extreme heterogeneity condition, $\beta^{h}$ was drawn from a Normal $\left(-3,2^{2}\right)$ distribution, which implies that $95 \%$ of elasticities lie in the range from 0.66 to -14.90 .

Incidence: Depending on the simulated values of the price parameters, we generate data for two scenarios. In the first, we explicitly allowed the decision to purchase in the category to be linked to the price sensitivity of the household, i.e., price-sensitive households are more likely to change their purchase timing decision based on price and promotion activity in the category. Consistent with results of earlier empirical analysis (Bucklin and Gupta 1992, Bucklin et al. 1998), the $\mathrm{CV}$ parameter was set to be 0.7 for the high pricesensitive households (price parameters smaller than

Table 1 Simulation Parameters

\begin{tabular}{llll}
\hline & \multicolumn{1}{c}{ Brand A } & Brand B & Brand C \\
\hline Utility Function & $2-\beta^{h} P_{A}$ & $1-\beta^{h} P_{B}$ & $0-\beta^{h} P_{C}$ \\
Regular Price & Uniform $(2.90,3.10)$ & Uniform $(2.40,2.60)$ & Uniform $(1.90,2.10)$ \\
Promoted Price & Uniform $(2.00,2.20)$ & Uniform $(1.90,2.10)$ & Uniform $(1.70,1.90)$ \\
Promotional Frequency & 1 out of 3 weeks & 1 out of 3 weeks & 1 out of 6 weeks \\
\hline
\end{tabular}


-3) while it was set to be 0.2 for the low price-sensitive households (price parameters greater than or equal to $-3)$. In the second condition, the category value response parameters were set equal to 0.2 for all households, implying the existence of price response heterogeneity without accompanying differences in purchase timing responsiveness.

\subsection{Simulation Results}

Condition 1. Moderate Price Response Heterogeneity/Purchase Timing Heterogeneity Present. Detailed parameter estimates obtained from fitting all three models in the above experimental condition are displayed in Table 2, along with the mean of the population parameter values used to generate the data.

According to Table 2, the coefficient for the sticker shock term in the NL-ST model is positive and significant even though the true model does not include a reference price term in the utility function. Additionally, in line with our predictions, the price coefficient for the NLST model has a smaller absolute value than its counterpart in the NL model $(2.329<2.583)$. These results confirm that, compared to a standard logit model, the introduction of the sticker shock term increases model flexibility, permitting the common coefficient $\beta_{c}$ to represent better the price response of the insensitive households. As is to be expected, parameter estimates are found to be seriously biased in the absence of heterogeneity correction.

In contrast, the posterior probability interval of the mean sticker shock coefficient obtained from the HBST model, i.e., the Bayesian equivalent of the confidence interval for the estimate of the population mean, contains the value zero. ${ }^{2}$ Thus, accounting for price response heterogeneity via household-specific price coefficients causes the spurious sticker shock effect to disappear. We also constructed the $95 \%$ probability interval of the sticker shock parameter estimated at the household level. Consistent with the data generation mechanism, this interval included zero for 399 out of the 400 households. As seen in Table 2, the HB-ST

\footnotetext{
${ }^{2}$ We estimated model parameters starting multiple chains of a hybrid of the Gibbs sampler and the Metropolis-Hastings algorithm with different starting values and found that, in all cases, the parameters were recovered by the model.
}

model also recovers the other parameters remarkably well.

Condition 2. Moderate Price Response Heterogeneity/Purchase Timing Heterogeneity Absent. In contrast to the results reported directly above, we find in this condition, that the coefficient for the sticker shock term is insignificant. Estimates of the sticker shock effect obtained from the NL-ST for this condition appear in the second row of Table 3 . This results implies that unaccounted for heterogeneity in price response may not always be sufficient to obtain a spuriously significant sticker shock effect.

Condition 3. Extreme Price Response Heterogeneity/ Purchase Timing Heterogeneity Absent. Parameter estimates from the NL-ST model, reported in the third row of Table 3, show that extreme price response heterogeneity is sufficient to cause biased estimates of the sticker shock coefficient, even when purchase timing heterogeneity is absent. However, the implied range of elasticities in this case is much larger than that reported in previous research (Tellis 1988). Therefore, within the empirically observable range of price response heterogeneity (i.e., the moderate case), differences in purchase timing responsiveness play an important role in determining the extent of bias.

Condition 4. Extreme Price Response Heterogeneity/ Purchase Timing Heterogeneity Present. The results, presented in the last row of Table 3, indicate that extreme price response heterogeneity in the choice decision, when combined with heterogeneity in purchase timing, can increase the bias in the sticker shock parameter.

Robustness Check. Extending Condition 1 to Include a Sticker Shock Term. Thus far, we have established the circumstances under which estimating the standard model results in a spuriously significant sticker shock coefficient, when the true model does not include a sticker shock term. We have also shown that, in this setting, the HB-ST model does not result in a spuriously significant coefficient.

To establish the ability of the HB-ST model to detect sticker shock effects when they are actually present, we extend the simulation so that the data generating model in Condition 1 includes a sticker shock term. 
CHANG, SIDDARTH, AND WEINBERG

Impact of Heterogeneity on Estimates of Sticker Shock Effects

Table 2 NL, NL-ST, and HB-ST Model Estimation Results on Simulated Data: Moderate Price Response Heterogeneity/ Purchase Timing Heterogeneity Present

\begin{tabular}{lcccc}
\hline Variable & True model $^{a}$ & $\mathrm{NL}$ & $\mathrm{NL}-\mathrm{ST}$ & $\mathrm{HB}-\mathrm{ST}$ \\
\hline BRAND A & 2.000 & 1.748 & 1.585 & 2.031 \\
& & $(26.492)^{b}$ & $(18.917)$ & {$[1.866,2.195]^{c}$} \\
BRAND B & 1.000 & 0.841 & 0.764 & 1.070 \\
& & $(17.595)$ & $(14.300)$ & {$[0.952,1.187]$} \\
PRICE & -3.102 & -2.583 & -2.329 & -3.082 \\
& & $(-29.886)$ & $(-19.589)$ & {$[-3.292,-2.873]$} \\
RPRICE-PRICE & 0.000 & - & 0.235 & 0.176 \\
& & & $(2.998)$ & {$[-0.030,0.382]$} \\
CONSTANT & \multirow{2}{*}{1.227} & 1.085 & 1.026 & 1.238 \\
& & $(11.216)$ & $(10.529)$ & {$[0.997,1.479]$} \\
CV & 0.475 & 0.566 & 0.630 & 0.448 \\
& & $(18.593)$ & $(15.272)$ & {$[0.394,0.502]$} \\
\hline
\end{tabular}

aMeans of household parameters used to generate the data.

${ }^{\mathrm{b}}$ Asymptotic $t$-statistics in parentheses.

c95\% probability intervals in brackets.

Table 3 NL-ST Model Estimation Results on Simulated Data: All Experimental Conditions

\begin{tabular}{lccc}
\hline $\begin{array}{l}\text { Price } \\
\text { Response } \\
\text { Heterogeneity }\end{array}$ & $\begin{array}{c}\text { Purchase } \\
\text { Timing } \\
\text { Heterogeneity }\end{array}$ & $\begin{array}{c}\text { Price } \\
\text { Parameter }\end{array}$ & $\begin{array}{c}\text { Sticker Shock } \\
\text { Parameter } \\
\text { (RPRICE-PRICE) }\end{array}$ \\
\hline MODERATE & PRESENT & -2.329 & 0.235 \\
$N\left(-3,1^{2}\right)$ & & $(-19.589)^{\mathrm{a}}$ & $(2.998)$ \\
MODERATE & ABSENT & -2.712 & 0.047 \\
$N\left(-3,1^{2}\right)$ & & $(-22.253)$ & $(0.597)$ \\
EXTREME & ABSENT & -2.332 & 0.175 \\
$N\left(-3,2^{2}\right)$ & & $(-19.963)$ & $(2.334)$ \\
EXTREME & PRESENT & -1.750 & 0.494 \\
$N\left(-3,2^{2}\right)$ & & $(-16.270)$ & $(7.272)$ \\
\hline
\end{tabular}

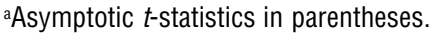

Thus brand utility is calculated according to Equation (1). In addition to the parameters and probability distributions already specified in Condition 1, we also draw a sticker shock coefficient for each household from a Normal $\left(0.5,0.3^{2}\right)$ distribution. ${ }^{3}$ The HB-ST

${ }^{3}$ To guide our selection of the distribution of sticker shock parameters, we examined the empirical results reported in previous re- model was estimated on the resulting simulated choice data. The estimated value, and associated $95 \%$ probability interval, for the estimated mean sticker shock parameter was found to be 0.630 [0.431, 0.83], which includes the true value of 0.5 . ${ }^{4}$ We also examined the $95 \%$ probability interval of the posterior distribution of the coefficients at the individual household level and found that, for all 400 households, these intervals included the true value used to generate the choices. In summary, these results confirm the ability of the HB-ST model to recover the sticker shock coefficient.

\section{Empirical Application}

To examine our predictions further, we conduct an empirical test using scanner panel data from the yogurt and ketchup categories. The HB-ST, NL-ST, and NL models are estimated on each dataset. Because the HBST model accounts for heterogeneity in all parameters across consumers and eliminates the source of sticker shock bias, we expect estimates of the sticker shock effect to be attenuated and to represent the true impact of reference prices on brand choice. Based on our earlier analysis, we expect the sticker shock coefficient from the NL-ST model to be positive and statistically significant.

\subsection{Data}

The data for this study are drawn from A. C. Nielsen scanner panel records in the yogurt and ketchup categories for households in Sioux Falls, South Dakota, for the period 1986-1988. The last 51 weeks of the data were used for model calibration, and the preceding 61 weeks were used for initializing model variables. Households qualified for inclusion in the sample if they made at least one grocery purchase every four weeks over the entire study period and made at least

search. We first identified those papers in which the model formulation, the price levels, and the manner in which prices and reference prices were operationalized were similar to those used in the simulation, e.g., Lattin and Bucklin (1989). To be conservative, the parameters of the distribution of the sticker shock coefficient were chosen so that the implied range was slightly larger than that reported in these papers, without overly affecting the implied price elasticities for this condition.

${ }^{4}$ The estimates from the NL-ST model were $0.644(0.493,0.795)$. 
one product purchase in both initialization and calibration periods. Out of the qualified households, 400 were randomly selected from each product category for model calibration (42,954 shopping trips and 3,852 purchases in the yogurt category, 44,631 shopping trips and 2,476 purchases in the ketchup category).

The top seven selling brands are included in the study. Together, these accounted for $84 \%$ (85\%) of category sales in dollars, and $89 \%(87 \%)$ in units for the yogurt and ketchup categories, respectively. These brands, their market shares by units, and average prices per ounce are given in Table 4.

\subsection{Model Variables}

The deterministic component of utility in the brand choice decision, for each alternative $i$, is specified as a function of the following household-specific and marketing mix variables:

$$
\begin{aligned}
\operatorname{BLOY}_{i}^{h}= & \text { within-household market share of brand } \\
& i \text { in the initialization period, } \\
\operatorname{LBP}_{i t}^{h}= & 1 \text { if } i \text { was the brand purchased on the pre- } \\
& \text { vious occasion; } 0 \text { otherwise, } \\
\text { PRICE }_{i t=} & \text { actual shelf price of brand } i \text { at time } t, \\
\operatorname{RPRICE}_{i t}^{h}= & \text { price of brand } i \text { on the last occasion on } \\
& \text { which household } h \text { made a purchase in } \\
& \text { the category, and }
\end{aligned}
$$

Table 4 Market Share and Average Price Yogurt and Ketchup Categories

\begin{tabular}{lcc}
\hline Brand & Market Share & Average Price per Ounce \\
\hline Yogurt & & \\
$\quad$ Yoplait & 0.19 & 10.1 cents \\
Weight Watchers & 0.12 & 7.7 cents \\
Dannon & 0.08 & 8.5 cents \\
Nordica & 0.29 & 6.7 cents \\
QC & 0.03 & 5.2 cents \\
W.B.B. & 0.17 & 5.4 cents \\
Private Label & 0.11 & 4.8 cents \\
Ketchup & & \\
Hunt's 32 0z. & 0.14 & 3.45 cents \\
Del Monte 32 0z. & 0.07 & 3.46 cents \\
Heinz 28 0z. & 0.23 & 4.52 cents \\
Heinz 32 0z. & 0.40 & 3.51 cents \\
Heinz 40 0z. & 0.06 & 4.83 cents \\
Heinz 64 0z. & 0.04 & 4.60 cents \\
Private Label & 0.06 & 2.76 cents \\
\hline
\end{tabular}

$\mathrm{PROMO}_{i t}=1$ if brand $i$ is on promotion at time $t ; 0$ otherwise.

The

above formulation accommodates cross-sectional heterogeneity in brand preference through the static brand loyalty measure and time-varying heterogeneity through the last purchase variable, $\mathrm{LBP}_{i t}^{h}$.

The deterministic component of utility for purchase incidence, $V_{t}^{h}$, is specified in terms of the following variables:

$$
\begin{aligned}
\mathrm{CR}^{h}= & \text { average weekly consumption rate for house- } \\
& \text { hold } h, \\
\mathrm{INV}_{t}^{h}= & \text { inventory estimate for household } h \text { at time } t, \\
& \text { and } \\
\mathrm{CV}_{t}^{h}= & \text { category value at time } t \text { for household } h .
\end{aligned}
$$

Consumption rate is computed as the total amount of product purchased by a household $h$ in the initialization period divided by the number of weeks in the period. The inventory variable, $\mathrm{INV}_{t}^{h}$, constructed according to the procedure described in Bucklin and Gupta (1992), is a measure of relative inventory within a household and is designed to capture the time-varying heterogeneity in incidence probabilities.

\subsection{Results and Discussion}

NL and NL-ST Models. Fit statistics and parameter estimates for these models are presented in Table 5. In both categories, the NL-ST model provides a better fit than the NL model and results in a sticker shock coefficient that is positive and significant. Also, as per our prediction, in both categories, the common price parameter $\beta_{c}$ in the NL-ST model is larger than the corresponding value in the NL model. Other parameters are very similar across the two models.

HB-ST Model. The superior fit of hierarchical Bayes random coefficients models has been documented elsewhere (Rossi et al. 1996, Allenby et al. 1998). Thus, we do not report extensive fit comparisons with the NL and NL-ST models, but confine our discussion to the parameter estimates. As is usual for Bayesian inference (e.g., Allenby and Lenk 1993, Allenby and Rossi 1998), we present the results in terms of the posterior distribution of the parameters, i.e., the posterior estimate of the mean $\mu$ and its associated $95 \%$ probability interval. Results are reported in Table 6. 
CHANG, SIDDARTH, AND WEINBERG

Impact of Heterogeneity on Estimates of Sticker Shock Effects

In both categories, the $95 \%$ probability interval for the mean sticker shock coefficient contains the value zero. Thus, properly accounting for price response heterogeneity mutes the impact of reference price and, for these datasets, the mean sticker shock effect disappears completely. In addition, another state-dependent variable, LBP, is insignificant in both categories supporting the notion that state dependence is attenuated by accounting for heterogeneity (Heckman 1981). Ainslie and Rossi (1998) report similar results for the ketchup and tuna categories.

An analysis of the sticker shock coefficients at the household level permits us to quantify the extent to which the sticker shock effect is prevalent. We constructed the $95 \%$ probability interval for each household-level posterior mean in both categories. We found that this interval contained zero for all but $9.75 \%$ of households in the yogurt category and $5.25 \%$ in the ketchup category, implying that only a very small proportion of households seem to experience sticker shock.

Alternative Formulations of Reference Price. Briesch et al. (1997) compare different approaches used to impute reference prices and conclude that an exponentially smoothed reference price measure based on a brand's price history provides the best fit. We reestimated the NL-ST model after operationalizing the reference price as $\mathrm{RP}_{i t}=\mathrm{RP}_{i(t-1)}(1-\alpha)+P_{i(t-1)} \alpha$. This measure provided a very slight improvement (about one log-likelihood point) in fit over the current measure, $\mathrm{RP}_{i t}=P_{i(t-1)}$, and $\alpha$ was estimated to be 0.72 (0.56) for the yogurt (ketchup) category. We then reestimated the HB-ST model using this measure of reference price, and found our results unchanged, i.e., the 95\% probability interval for the mean sticker shock coefficient still contained the value zero while the other parameter values were virtually the same. ${ }^{5}$

Kalwani et al. (1990) have expanded on the basic sticker shock model and captured loss aversion via separate coefficients for gain and loss terms. We first estimated this version of the NL-ST model and found,

${ }^{5}$ Estimated parameter values for the sticker shock coefficient were $0.092[-0.037,0.221]$ and $0.156[-0.091,0.403]$ in the yogurt and ketchup categories, respectively.
Table 5 NL and NL-ST Parameter Estimates and Fit Statistics ${ }^{a}$

\begin{tabular}{|c|c|c|c|c|}
\hline \multirow[b]{2}{*}{ Variable } & \multicolumn{2}{|c|}{ Yogurt } & \multicolumn{2}{|c|}{ Ketchup } \\
\hline & NL & NL-ST & NL & NL-ST \\
\hline \multicolumn{5}{|l|}{ Brand Choice } \\
\hline BLOY & $\begin{array}{c}1.701 \\
(24.898)^{b}\end{array}$ & $\begin{array}{c}1.694 \\
(24.608)\end{array}$ & $\begin{array}{c}2.540 \\
(22.804)\end{array}$ & $\begin{array}{r}2.520 \\
(22.654)\end{array}$ \\
\hline LBP & $\begin{array}{c}1.230 \\
(31.054)\end{array}$ & $\begin{array}{c}1.264 \\
(30.913)\end{array}$ & $\begin{array}{c}0.776 \\
(14.170)\end{array}$ & $\begin{array}{r}0.826 \\
(14.631)\end{array}$ \\
\hline PRICE & $\begin{array}{c}-0.365 \\
(-12.769)\end{array}$ & $\begin{array}{c}-0.279 \\
(-8.213)\end{array}$ & $\begin{array}{c}-1.266 \\
(-17.941)\end{array}$ & $\begin{array}{r}-1.065 \\
(-12.104)\end{array}$ \\
\hline $\begin{array}{l}\text { RPRICE - } \\
\text { PRICE }\end{array}$ & & $\begin{array}{c}0.126 \\
(4.394)\end{array}$ & & $\begin{array}{c}0.299 \\
(4.101)\end{array}$ \\
\hline PROMO & $\begin{array}{c}1.464 \\
(21.706)\end{array}$ & $\begin{array}{c}1.447 \\
(21.337)\end{array}$ & $\begin{array}{c}1.960 \\
(26.510)\end{array}$ & $\begin{array}{c}1.937 \\
(26.349)\end{array}$ \\
\hline \multicolumn{5}{|c|}{ Purchase Incidence } \\
\hline CONSUME & $\begin{array}{c}0.068 \\
(34.628)\end{array}$ & $\begin{array}{c}0.068 \\
(34.963)\end{array}$ & $\begin{array}{c}0.122 \\
(23.281)\end{array}$ & $\begin{array}{c}0.122 \\
(23.334)\end{array}$ \\
\hline INVENTORY & $\begin{array}{c}-0.073 \\
(-2.676)\end{array}$ & $\begin{array}{c}-0.074 \\
(-2.675)\end{array}$ & $\begin{array}{c}-1.382 \\
(-21.295)\end{array}$ & $\begin{array}{r}-1.383 \\
(-21.265)\end{array}$ \\
\hline CV & $\begin{array}{c}0.429 \\
(12.456)\end{array}$ & $\begin{array}{c}0.418 \\
(12.245)\end{array}$ & $\begin{array}{c}0.382 \\
(11.680)\end{array}$ & $\begin{array}{c}0.379 \\
(13.511)\end{array}$ \\
\hline Log-likelihood & -16924.600 & -16914.391 & -11949.074 & -11940.608 \\
\hline
\end{tabular}

${ }^{a}$ Brand-specific constants are not presented.

${ }^{b}$ Asymptotic $t$-statistics in parentheses.

in both categories, that the difference in magnitude between the gain and loss coefficients was statistically insignificant-providing no evidence for loss aversion. ${ }^{6}$ The same results held for the HB-ST model.

Relationship Between Price Sensitivity and Responsiveness to Category Value. Previously in the paper, we had argued that estimates of the sticker shock effect can be adversely impacted if households that are price-sensitive in the choice decision are also more responsive to price and promotion in the category purchase decision. To provide support for this argument, we can examine the correlation between a household's CV parameter and its price parameter. Therefore, during estimation, we also simultaneously

${ }^{6}$ The ratio of the loss coefficient $\left(\beta_{l}\right)$ to the gain coefficient $\left(\beta_{g}\right)$ was also insignificant. The variance of the ratio $\left(\lambda=\beta_{l} / \beta_{g}\right)$ is given by Kramer's Theorem, i.e., $\left(\partial \lambda / \partial \beta_{i}\right)^{\prime} \Sigma\left(\partial \lambda / \partial \beta_{i}\right)$ where $\Sigma$ is the variance covariance matrix for the estimated parameters $\beta_{i}$ for $\mathrm{i}=1$, g. 
CHANG, SIDDARTH, AND WEINBERG

Impact of Heterogeneity on Estimates of Sticker Shock Effects

Table 6 HB-ST Parameter Estimates ${ }^{a}$

\begin{tabular}{lcc} 
Variables & $\mu$ (Yogurt) & $\mu$ (Ketchup) \\
\hline Brand Choice & & \\
BLOY & 3.387 & 4.637 \\
& {$[3.087,3.688]^{b}$} & {$[4.278,4.995]$} \\
LBP & -0.017 & -0.209 \\
& {$[-0.196,0.162]$} & $(-0.427,0.009)$ \\
PRICE & -0.597 & -1.887 \\
& {$[-0.705,-0.490]$} & {$[-2.036,-1.736]$} \\
RPRICE - & 0.046 & 0.125 \\
PRICE & {$[-0.089,0.181]$} & {$[-0.099,0.349]$} \\
PROMO & 1.628 & 2.505 \\
& {$[1.338,1.919]$} & {$[2.187,2.823]$} \\
Purchase Incidence & & \\
CONSUME & 0.091 & 0.143 \\
& {$[0.013,0.170]$} & {$[0.055,0.230]$} \\
INVENTORY & -0.864 & -3.174 \\
& {$[-1.054,-0.675]$} & {$[-3.469,-2.878]$} \\
CV & 0.368 & 0.311 \\
& {$[0.251,0.485]$} & {$[0.208,0.413]$} \\
\hline
\end{tabular}

aBrand-specific constants are not presented.

${ }^{\circ} 95 \%$ probability interval in brackets.

sampled posterior estimates of the coefficient from a regression of household $\mathrm{CV}$ parameters on the price parameters. The posterior estimate of the regression coefficient, and its 95\% probability interval, was $-0.185[-0.271,-0.098]$ in the yogurt category and $-0.078[-0.113,-0.043]$ in the ketchup category. These results confirm that, in both categories, greater responsiveness to category value is associated with higher price sensitivity in the choice decision.

\section{Conclusion}

The reference price construct, and its role in the brand choice decision, has been extensively investigated by researchers in marketing using secondary data from scanner panels. Consumer heterogeneity also has a rich modeling tradition in the marketing literature, and several papers have highlighted its important influence on parameter estimates obtained from choice and incidence models. However, most previously reported results on reference price effects have been based on models that have not adequately accounted for response heterogeneity.
The current work studies the impact of unaccounted-for heterogeneity on estimates of the sticker shock effect. The analysis reveals two conditions which influence the extent of upward bias in the sticker shock coefficient. First, the estimated model incompletely accounts for true differences in price sensitivities among consumers. Second, heterogeneity in purchase timing systematically varies with the underlying price responsiveness. Based on this analysis, several predictions about the nature of parameter bias are made, and confirmed via a simulation study. Results from the study also show that while unaccounted for price response heterogeneity can, in itself, result in biased estimates of the sticker shock effect, the implied range of price response variation is much larger than observed in practice.

A hierarchical Bayes version of the nested logit model, which captures heterogeneity in a continuous random effects framework, is formulated and estimated on scanner panel data from two product categories. In contrast to the standard nested logit model with a sticker shock term, that does not account for price response heterogeneity, the $95 \%$ probability interval of the mean sticker shock parameter in the HBST model includes the value zero. For both these data sets, therefore, the results do not support the existence of a sticker shock effect as operationalized in previous research. Consistent with our explanation of the underlying cause of bias, greater responsiveness to category value is associated with higher price sensitivity in the choice decision. Overall, these results provide strong support for our theory of the determinants of sticker-shock bias and underscores the important role played by purchase timing heterogeneity.

Our results complement those of Bell and Lattin (1998), who focus on the reference dependent formulation of reference price. Their explanation for bias is consistent with our analysis. Thus, in their work, gains and losses mimic the pattern of price heterogeneity of the underlying segments and play the same role as the sticker shock term does in our model. Our empirical work in two product categories (yogurt and ketchup) shows that accounting for price response heterogeneity results in an insignificant sticker shock coefficient. Their results show that loss aversion is significantly 
attenuated in all twelve categories that they analyze, disappearing in five of them.

Recent work in the marketing literature has used reference price effects to derive optimal promotion and pricing policies (Koppalle et al. 1996, Greenleaf 1995). Accurate estimates of the size and prevalence of reference price effects are critical to the development of these strategies; our research highlights the importance of accounting for consumer heterogeneity in obtaining such estimates.

Future research could take several directions. Laboratory and survey work could be used to uncover the mechanisms that consumers actually use to form reference prices in different product categories. Besides providing a useful framework to understand the construct, this research may suggest better heuristics for formulating reference price measures to be used in studies using secondary data sources. Another fruitful area for research is modeling the structural (or model) uncertainty associated with how consumers respond to reference prices. A Bayesian model averaging approach (Draper 1995) could be implemented via a flexible model in which some consumers responded to a common category level reference price (via the reference dependent model) while others used a brandspecific reference price (the sticker shock model). Still others might not respond to reference prices at all. Such a model could potentially provide better insights than can be obtained by using any one of the models individually.

The objective of this research was to describe the mechanism by which response heterogeneity could bias estimates of the sticker shock effects of reference price. Our work points out some of the limitations of previous studies and shows that considerable additional analysis, employing a more complete treatment of response heterogeneity, perhaps over multiple categories, will be needed to describe accurately the formation and role of reference prices in brand choice. ${ }^{7}$

\footnotetext{
${ }^{7}$ The paper has benefited from comments by Asim Ansari, David Bell, Randy Bucklin, seminar participants at a joint workshop of the University of British Columbia and the University of Washington, the editor, area editor, and two anonymous reviewers of this journal. The authors thank the A. C. Nielsen Company for providing the data used in this study. This research was partially supported by a research grant from the Social Sciences and Humanities Research Council of Canada awarded to the second author.
}

\section{Appendix A: Priors}

We specify a multivariate normal prior over household parameters, $\theta^{h}$ as follows:

$$
\boldsymbol{\theta}^{h} \sim \operatorname{MVN}(\boldsymbol{\mu}, \boldsymbol{\Sigma})
$$

Following Gelfand et. al. (1990), hyper-priors over population parameters, $\mu$ and $\Sigma$ are specified as:

$$
\begin{gathered}
\boldsymbol{\mu} \sim \operatorname{MVN}(\boldsymbol{\eta}, \mathbf{C}), \\
\boldsymbol{\Sigma}^{-1} \sim W\left((\rho \mathbf{R})^{-1}, \rho\right),
\end{gathered}
$$

where $\boldsymbol{\eta}=0, \mathbf{C}^{-1}=0, R=15 \mathbf{I}$, and $\rho$, the degrees of freedom for the Wishart distribution, is fixed at 16 (a number of parameters plus one). These parameters, therefore, represent diffuse (noninformative) but proper distributions.

\section{Appendix B: Full Conditionals and Simulations}

B.1. Generating $\boldsymbol{\theta}^{h(m+1)}$

Given the simulated $\boldsymbol{\mu}^{(m)}$ and $\boldsymbol{\Sigma}^{-1(m)}$, we sample household-specific $\boldsymbol{\theta}^{h(m+1)}$ from the following full conditional distribution:

$$
p\left(\theta^{h(m+1)} \mid \boldsymbol{\mu}^{(m)}, \Sigma^{(m)}\right) \propto L\left(\theta^{h(m+1)}\right) \phi\left(\theta^{h(m+1)} \mid \boldsymbol{\mu}^{(m)}, \Sigma^{(m)}\right),
$$

where

$$
\begin{aligned}
L\left(\boldsymbol{\theta}^{h(m+1)}\right)= & \Pi_{t=1}^{T^{h}}\left[P_{t}^{h}(\text { inc })^{\delta_{t}^{h}}\left(1-P_{t}^{h}(\text { inc })\right)^{1-\delta_{t}^{h}}\right. \\
& \left.\Pi_{i}\left[P_{t}^{h}(i \mid \text { inc })\right]^{\delta_{t h}^{h}}\right]
\end{aligned}
$$

$\delta_{i t}^{h}=1$ if household $h$ bought brand $i$ on a store visit at time $t$, and zero otherwise, $\delta_{t}^{h}=\Sigma_{i} \delta_{i t}^{h}$ and $\phi(\cdot \mid \cdot)$ is a multivariate normal density.

This posterior distribution is known only up to a normalizing constant since it is a product of a normal prior and logit likelihood. Therefore, we adopt the random walk version of the MetropolisHastings algorithm (Tierney 1994, Chib and Greenberg 1995, Carlin and Chib 1995, Green 1995). In this version, given the previous draw $\boldsymbol{\theta}^{h(m)}$ in the chain, a candidate draw $\boldsymbol{\theta}^{h(c)}$ is simulated as:

$$
\boldsymbol{\theta}^{h(c)}=\boldsymbol{\theta}^{h(m)}+\mathbf{u},
$$

where $\mathbf{u} \sim \operatorname{MVN}(0, \boldsymbol{V}), \boldsymbol{V}$ is a prespecified matrix. ${ }^{8}$ Then $\boldsymbol{\theta}^{h(c)}$ is accepted as $\boldsymbol{\theta}^{h(m+1)}$ in the chain with the following probability:

$$
\min \left[\frac{L\left(\boldsymbol{\theta}^{h(c)}\right) \phi\left(\boldsymbol{\theta}^{h(c)} \mid \boldsymbol{\mu}^{(m)}, \mathbf{\Sigma}^{(m)}\right)}{L\left(\boldsymbol{\theta}^{h(m)}\right) \phi\left(\boldsymbol{\theta}^{h(m)} \mid \boldsymbol{\mu}^{(m)}, \mathbf{\Sigma}^{(m)}\right)^{\prime}}, 1\right] .
$$

Therefore, we do not need to know the normalizing constant in simulating draws. If the candidate draw is rejected, we set $\boldsymbol{\theta}^{h(m+1)}=$ $\boldsymbol{\theta}^{h(m)}$.

${ }^{8}$ In our empirical application, following Chiang et al (1998), we let $V$ be twice the negative inverse of the observed information matrix of the NL-ST likelihood. However, any other suitable choice would also suffice as long as it ensures acceptance rates between $25 \%$ and 50\% (see Chib and Greenberg 1995). 


\section{B.2. Generating $\boldsymbol{\mu}^{(m+1)}$}

Given the simulated $\left\{\boldsymbol{\theta}^{h(m)}\right\}$ across households and $\boldsymbol{\Sigma}^{-1(m)}, \boldsymbol{\mu}^{(m+1)}$ is drawn from the following full conditional distribution (a posterior multivariate normal).

$$
\begin{aligned}
& \left.p\left(\boldsymbol{\mu}^{(m+1)} \mid\left\{\boldsymbol{\theta}^{h(m)}\right\}, \boldsymbol{\Sigma}^{(m)}, \boldsymbol{\eta}\right), \mathbf{C}\right) \\
& \quad=\operatorname{MVN}\left[\mathbf{D}\left(H \boldsymbol{\Sigma}^{-1(m)} \overline{\boldsymbol{\theta}}+C^{-1} \boldsymbol{\eta}, \mathbf{D}\right]\right.
\end{aligned}
$$

where $\mathbf{D}=\left(H \boldsymbol{\Sigma}^{-1(m)}+\mathbf{C}^{-1}\right)^{-1}, \bar{\theta}=1 / H \sum_{h=1}^{H} \boldsymbol{\theta}^{h(m)}$ and $H$ is the total number of households.

\section{B.3. Generating $\Sigma^{-1(m+1)}$}

Given the simulated $\left\{\boldsymbol{\theta}^{h(m)}\right\}$ across households and $\boldsymbol{\mu}^{(m)}$, we simulate $\boldsymbol{\Sigma}^{-1(m+1)}$ from the following full conditional distribution (a posterior Wishart):

$$
\begin{aligned}
& p\left(\boldsymbol{\Sigma}^{-1(m+1)} \mid\left\{\boldsymbol{\theta}^{h(m)}\right\}, \boldsymbol{\mu}^{(m)}, \rho, \mathbf{R}\right)= \\
& W\left[\left[\sum_{h=1}^{H}\left(\boldsymbol{\theta}^{h(m)}-\boldsymbol{\mu}^{(m)}\right)\left(\boldsymbol{\theta}^{h(m)}-\boldsymbol{\mu}^{(m)}\right)^{\prime}+\rho \mathbf{R}\right],-1 H+\rho\right] .
\end{aligned}
$$

\section{References}

Ainslie, Andrew, Peter E. Rossi 1998. Similarities in choice behavior across product categories. Marketing Sci. 17 (2) 91-106.

Allenby, Greg M., Neeraj Arora, James L. Ginter 1998. On the heterogeneity of demand. J. Marketing Res. 35 (August) 384-389.

—, Peter J. Lenk 1994. Modeling household purchase behavior with logistic normal regression. J. Amer. Statist. Assoc. 89 12181231.

— neity. J. Econometrics 89 (1-2) 57-78.

Arora, Neeraj, Greg M. Allenby, James L. Ginter 1998. A hierarchical Bayes model of primary and secondary demand. Marketing Sci. 17 (1) 29-44.

Bell, David R., James M. Lattin 1998. Looking for loss aversion in scanner panel data: the confounding effect of price-response heterogeneity. Wharton Marketing Department Working Paper No. 98-032.

— Randolph E. Bucklin 1999. The role of internal reference points in the category purchase decision. Wharton Marketing Department Working Paper No. 98-025.

Briesch, Richard A., Lakshman Krishnamurthi, Tridib Mazumdar, S. P. Raj 1997. A comparative analysis of reference price models. J. Consumer Res. 24 202-214.

Bucklin, Randolph E., Sunil Gupta 1992. Brand choice, purchase incidence, and segmentation: an integrated modeling approach. J. Marketing Res. 24 (May) 201-215.

,-- S. Siddarth 1998. Determining segmentation in sales response across consumer purchase behaviors. J. Marketing Res. 35 (May) 189-197.

Carlin, B. P., S. Chib 1995. Bayesian model choice via Markov chain Monte Carlo methods. J. Royal Statis. Soc. Series B 57(3) 473-484.

Chiang, Jeongwen, Siddartha Chib, Chakravarthi Narasimhan 1998. Estimation of a heterogeneous consideration set brand choice model using scanner panel data. J. Econometrics. 89 (MarchApril) 223-248.

Chib, S., E. Greenberg 1995. Understanding the Metropolis-Hastings algorithm. Amer. Statistician 49 327-335.

Chintagunta, P. 1992. Estimating a multinomial probit model of brand choice using the method of simulated moments. Marketing Sci. 11(4) 386-407.

- 1993. Investigating purchase incidence, brand choice and purchase quantity decisions of households. Marketing Sci. 12(2) 184-208.

Draper, David 1995. Assessment and propagation of model uncertainty. J. Royal Statist. Soc. Series B 57(1) 45-97.

Gelfand, A. E., A. F. M. Smith 1990. Sampling based approaches to calculating marginal densities. J. Amer. Statist. Assoc. 85 398409.

— S. E. Hills, A. Racine-Poon, A. F. M. Smith 1990. Illustration of Bayesian inference in normal data models using Gibbs sampling. J. Amer. Statist. Assoc. 90 972-985.

Geman, S., D. Geman 1984. Stochastic relaxation, Gibbs distributions and the Bayesian restoration of images. IEEE Trans. on Pattern Analysis and Machine Intelligence 6 721-741.

Gönül, F., Kannan Srinivasan 1992. Modeling multiple sources of heterogeneity in multinomial logit models: methodological and managerial issues. Marketing Sci. 12(3) 213-229.

Green, Peter J. 1995. Reversible jump Markov chain Monte Carlo computation and Bayesian model determination. Biometrika 82(4) 711-732.

Greenleaf, E. A. 1995. The impact of reference price effects on the profitability of price promotions. Marketing Sci. 14(1) 82-104.

Gupta, Sunil 1988. Impact of sales promotions on when, what, and how much to buy. J. Marketing Res. 25 (November) 342-355.

Hardie, B. G. S., E. J. Johnson, P. S. Fader 1993. Modeling loss aversion and reference dependence effects on brand choice. Marketing Sci. 12 (4) 378-394.

Heckman, J. J. 1981. Heterogeneity and state dependence. S. Rosen, Ed. Studies in Labor Markets. University of Chicago Press, Chicago IL.

Helsen, K., David Schmittlein 1993. Analyzing duration times in marketing: evidence for the effectiveness of hazard rate models. Marketing Sci. 12 (4) 395-414.

Helson, H. 1964. Adaptation Level Theory. Harper and Row, New York.

Jain, D. C., N. J. Vilcassim 1991. Investigating household purchase timing decisions: a conditional hazard function approach. Marketing Sci. 10 (1) 1-23.

Kahneman, D., A. Tversky 1979. Prospect theory: an analysis of decision under risk. Econometrica 47 (March) 263-291.

Kalwani, Manohar U., Chi Kin Yim, Heikki J. Rinne, Yoshi Sugita 1990. A price expectations model of customer brand choice. J. Marketing Res. 27 (August) 251-262.

Kalyanaram, Gurumurthy, J. D. C. Little 1994. An empirical analysis of latitude of price acceptance in consumer package goods. J. Consumer Res. 21 (December) 408-418.

Kopalle, P. K., Ambar G. Rao, Joao L. Assuncao 1996. Asymmetric 
CHANG, SIDDARTH, AND WEINBERG

Impact of Heterogeneity on Estimates of Sticker Shock Effects

reference price effects and dynamic pricing policies. Marketing Sci. 15(1) 60-85.

Lattin, J. M., R. E. Bucklin 1989. Reference effects of price and promotion on brand choice behavior. J. Marketing Res. 26 (August) 299-310.

Mayhew, G. E., Russell S. Winer 1992. An empirical analysis of internal and external reference prices using scanner data. J. Consumer Res. 19 (June) 62-70.

McCulloch, R., Peter Rossi 1994. An exact likelihood approach to analysis of the MNP model. J. Econometrics 64 207-240.

Rajendran, K. N., G. J. Tellis 1994. Contextual and temporal components of reference price. J. Marketing 58 (January) 22-34.

Raftery, A. E., S. M. Lewis 1995. How many iterations of the Gibbs sampler. J. M. Bernard, J. O. Berger, A. P. Davis, A. F. M. Smith, eds. Bayesian Statistics 4. Oxford University Press, Oxford, UK.
Rossi, Peter E., Robert E. McCulloch, Greg M. Allenby 1996. The value of purchase history data in target marketing. Marketing Sci. 15(4) 321-340.

Sherif M., D. Taub, C. I. Hovland 1958. Assimilation and contrast effects of anchoring stimuli on judgments. J. Experimental Psych. 55(2) 150-155.

Tellis, Gerard J. 1988. The price elasticity of selective demand: a meta-analysis of econometric models of sales. J. Marketing Res. 25 (November) 331-341.

Tierney, L. 1994. Markov chains for exploring posterior distributions. Ann. Statist. 22 1701-1762.

Winer, R. S. 1986. A reference price model of brand choice for frequently purchased products. J. Consumer Res. 13 (September) 250-256.

This paper was received June 4, 1998, and has been with the authors 3 months for 2 revisions; processed by Wagner Kamakura. 